\title{
Efficient spatial-temporal chaotic mixing in microchannels
}

\author{
Xize Niu and Yi-Kuen Lee \\ Department of Mechanical Engineering, Hong Kong University of Science and Technology, \\ Hong Kong, People's Republic of China \\ E-mail: meyklee@ust.hk
}

Received 5 November 2002, in final form 3 March 2003

Published 11 April 2003

Online at stacks.iop.org/JMM/13/454

\begin{abstract}
A chaotic micro mixer with multiple side channels is designed and investigated, in which fluid can be stirred by pumps through the side channels. By stretching and folding fluid in the main and side channels, chaotic mixing can be achieved. A simple mathematic model is derived to understand the movement of particles in the microchannel. Spatial trajectories of fluid particles are projected to Poincaré sections by mapping. The route from the quasi-period to chaos is revealed to be destruction of KAM curves and shrinkage of the quasi-periodic areas. Lyapunov exponents (LE) are used as the mixing index and the criteria to evaluate the chaotic behavior of the system. We found that LE is closely related to the amplitude and frequency of stirring and can be used to optimize our design and operation. From the relationship of LE and striation thickness, the minimal mixing length required can be estimated, which is much shorter than that needed in passive mixer design.
\end{abstract}

\section{Introduction}

Over the past few years, numerous microfluidic devices have been designed and widely used in biology and biotechnology [1-7]. A typical kind of microfluidic device for mixing and convection is the microchannel with different geometric designs. These microchannels need much smaller volumes of biochemical reactants than analyzers in traditional technology and their performance is more efficient due to their enhanced heat and mass transfer properties. Another advantage is the ease with which fluid flow can be controlled by applying an electric field, pressure gradient, geometry design of the channel or by a combination of these methods.

But as we know, full mixing in microchannels is difficult to achieve because flows of common liquids at practical pressure in microchannels are characterized by low Reynolds number $(R e)$, generally less than 100 . The flow is restricted to the laminar flow regime and there is no turbulence. If we only rely on molecular diffusion to ensure a completely mixed outlet stream, the mixing channel has to be extended to extremely long.

To achieve a faster mixing, several passive micromixers have been developed and studied, such as the T-type [3], L-shaped [6], serpentine pipe [8], flow splitting [9] and so on.
It was shown that altering the inlet angle in the T-type device does not significantly improve mixing. For the L-shaped conduits, the bend can induce center-rotating vortices. Such vortices may assist the stirring process and enhance mixing. Unfortunately, at low values of $R e$, the bend-induced vortices decay before they have an opportunity to significantly stir the fluid. In order to increase the effectiveness of those vortices, it is necessary to use a large number of bends, in a plane or out of plane. Splitting different flows and rearranging them in alternating thin laminae will significantly decrease mixing time compared with pure diffusion because a subdivision of each stream into $n$ laminae can lead to $n^{2}$-times faster mixing. Except for the need of being fabricated delicately to arrange all the splitting and confluence in a chip, these structures are usually not very efficient either in time or in space. Usually a very long channel is needed to allow molecular diffusion to complete mixing.

Successive stretching and folding of fluid elements in microchannels can dramatically decrease striation thickness, which are building blocks of fluid mixing besides molecular diffusion [10]. Therefore, in order to understand and optimize the fluid deformation during the mixing process, it is necessary to understand the mechanism of fluid stretching and folding, and evaluate them in a quantitative manner. In the past 
Efficient spatial-temporal chaotic mixing in microchannels

two decades, it has been demonstrated that chaotic mixing is associated with such stretching and folding of fluid described in the Lagrangian representation $[10,11]$. For an unsteady flow with more than 2 degrees of freedom, which is usually an unintegrable Hamiltonian system, chaos is almost universal. Global bifurcation theories are used to analyze homoclinic and heteroclinic bifurcations and the breaking up of the Kolmogorov Arnold Moser (KAM) curves. Different kinds of mapping and Poincaré sections are used to simplify the original systems by reducing their dimensions. Also, Lyapunov exponents $[12,13]$ that are equivalent to the long time average of a specific fluid stretching rate have been used to evaluate mixing efficiency [10].

For a channel flow, to increase the mixing rate and to reduce the mixing length, there must exist transverse components of the flow velocity that can stretch and fold volumes of fluid over the cross section of the channel, namely, inducing off-axis or lateral transport within the channel. This is nontrivial, especially in the typical cases that require mixing two kinds of liquids in a microchannel. If the interface cannot be stirred sufficiently to be off-axis, no rapid mixing can be expected. A staggered herringbone mixer and a series of ablated wells in the channel are designed to induce lateral transports and generate chaotic flows $[1,2,4,8]$.

For these passive micromixers, a channel of considerable length is still needed; moreover, how to choose the appropriate parameters to optimize the system is also a tricky problem. It is quite difficult to analyze theoretically the velocity fields of flow passing through these complex asymmetry structures. Even for CFD simulation using modern powerful computers, it is a challenging task.

To facilitate rapid mixing, an active design can also be used so that fluid in the channel can be stirred by sources of pressure perturbations [5]. Within this channel, lateral transport can be controlled by various amplitudes and frequencies of perturbation. A multiple side channel design was advocated in $[14,19]$ with a working principle of reorienting the lamination of the flow. An alternating on-off mechanism of each stream flowing into the beginning channel can produce crescent-shaped interfaces in the downstream regions of the main channel [20], which behaves as the active mixers with only one pair of side channels [5]. But no chaotic dynamical analyses of these kinds of active mixers appear so far. In this context, we designed a new micromixer with multiple side channels, with which we can obtain chaotic mixing for almost all the fluid elements in the channel by choosing a group of optimized parameters. Mathematically, this system is also very simple. Moreover, we deduced a suitable mapping of this mixer in order to generate useful Poincaré section. With the help of this section, how the particles' movements vary from quasi-periodic to chaos, and rupture of the KAM curves can be shown explicitly. Besides qualitative analysis, Lyapunov exponents (LE) are used as a mixing index for the proposed micromixer. By calculating the LE with different parameters per unit area of fluid in the beginning channel, we can obtain a complete picture of chaotic dynamic information of the system, which can be used to optimize the micromixer design.

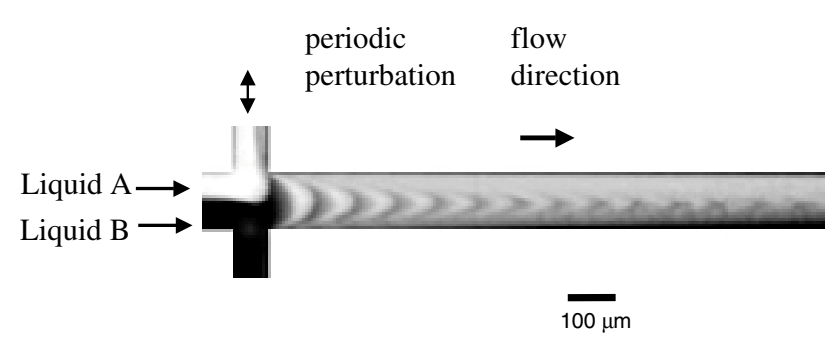

(a)

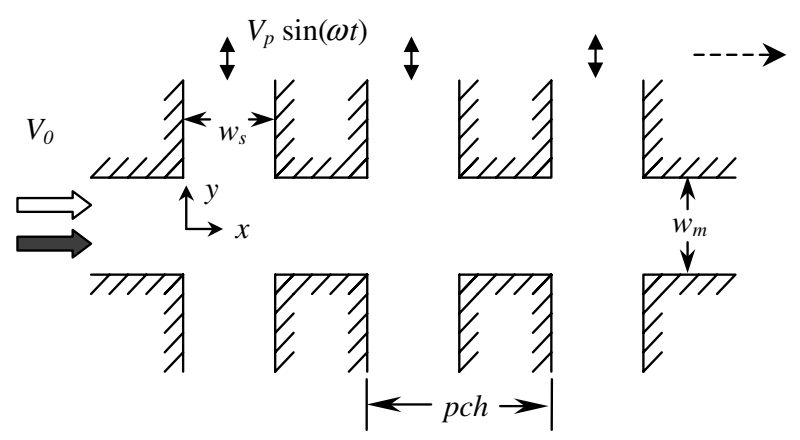

(b)

Figure 1. Model of a chaotic mixer with multiple side channels. (a) Experimental results of mixing in the device with one pair of side channels. The pressure perturbations induce lobe-like distortions of the interface and facilitate rapid mixing [5]. (b) Schematic showing the new mixer with multiple side channels.

\section{Model of the system}

\subsection{Physical model}

By using standard MEMS technology, a micromixer with one main channel and one pair of side channels can be fabricated as shown in figure 1 $(a)$ [5]. Two kinds of fluids are injected from the inlet with the same flow rate. Each pair of side channels is connected to a pair of high and low pressure sources via a computer controlled high-speed solenoid valve to achieve a kind of source-sink mechanism. Therefore, timeperiodic pressure perturbations are applied transversally to the main stream. Then we designed the mixer with multiple side channels as schematically shown in figure $1(b)$. For simplicity, we set every perturbation to the same phase and amplitude.

Because the angle of the two inlet channels has little influence on the mixing of the two fluids in the main channel [3], we can assume that at the beginning part of the main channel, the interface between the liquids lies right in the middle of the channel, and no off-axis advection appears at this stage.

\subsection{Mathematical model}

Since the flow velocity in the micro-channel is very slow with a small Reynolds number, the micromixer works in a regime of creeping flow. The Navier-Stokes equations can be linearized to the equations for a Stokes flow, and the velocity field at the intersection of the main and side channels should be the linear superposition of their original flow patterns. The position vector $(x, y)$ of each particle in the channel is governed by the 
following ordinary differential equations:

$\dot{x}=\left\{\begin{array}{lll}v_{0}\left[1-\left(y / w_{m 2}\right)^{2}\right] & \text { if } & |y|<w_{m 2} \\ 0 & \text { if } & |y|>w_{m 2}\end{array}\right.$

$\dot{y}=\left\{\begin{array}{lll}v_{p}\left\{1-\left[\left(x-x_{c}\right) / w_{s 2}\right]^{2}\right\} \sin (\omega t) & \text { if } & |x|<x_{p}+w_{s} \\ 0 & \text { if } & |x|>x_{p}+w_{s}\end{array}\right.$

where $x_{c}=x_{p}+w_{s 2}$ and $x_{p}=p c h * \operatorname{fix}(x / p c h)$. pch is the length of each unit of the main channel; $w_{m}$ is the span width of the main channel; $w_{s}$ is the span width of the side channel; $v_{0}$ is the initial velocity of fluid particles on the interface line in the beginning channel; and $v_{p}$ and $\omega$ are the amplitude and angle frequency of perturbation, respectively. After normalization, the parameters in equations (1) are: $p c h=4 ; w_{m}=2 ; w_{s}=2 ; w_{m 2}=w_{m} / 2 ; w_{s 2}=w_{s} / 2$; $v_{0}=1 ; v_{p}=1 ; \omega=1$. For a fixed structure, $v_{p}$ and $\omega$ can be adjusted to achieve optimal mixing, and be treated as bifurcation parameters in the following analysis.

Once the velocity field has been described, the particle trajectories can be easily calculated by integrating equations (1). Although molecular diffusion has not been taken into account in this model, it can be used as a good approximation [14].

\subsection{Dynamic analysis}

A traditional chaotic system generally has at least two basic ingredients. First, the system is sensitive to initial conditions, that is, nearby orbits from the initial points in the basin of the chaotic attractor will separate exponentially in time (stretching), therefore, one or more Lyapunov exponents should be positive. Second, the motion or the mapping of motion should be bounded, so that the exponential separation does not simply result in a smooth expansion to infinity as the vector field of a saddle point of a linear system. This means the separating orbits must eventually come close together again (folding).

If $v_{p}=0$, the fluid will remain in the main channel and (1) is an integrable Hamiltonian system with the Hamiltonian function $H=y-y^{3} / 3+$ const. This kind of system cannot have asymptotic equilibrium positions and asymptotically stable limit cycles in the phase space, therefore the system cannot be chaos.

If $v_{p} \neq 0$ (and $\omega \neq 0$ from the viewpoint of practical operation), (1) becomes a two-dimensional non-autonomous system, and also a nonintegrable Hamiltonian system. Chaos may occur and fluid elements may be stretched and folded by the transverse perturbation provided through side channels. The analysis of vector field in each unit of the mixer can help us understand the dynamics in the channel. Each unit can be divided into four areas as shown in figure 2. The motions of the particles in areas 1, 2 and 3 are very simple according to (1a). While in area 4 , the governing equations are

$$
\left\{\begin{array}{l}
\dot{x}=v_{0}\left[1-\left(y / w_{m 2}\right)^{2}\right] \\
\dot{y}=v_{p}\left\{1-\left[\left(x-x_{c}\right) / w_{s 2}\right]^{2}\right\} \sin (\omega t) .
\end{array}\right.
$$

Equation (2) has four equilibrium points (EP), and they are exactly the corners of the intersection of the main and side

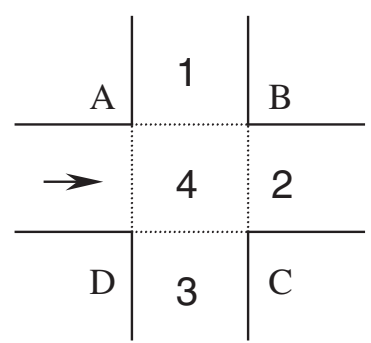

Figure 2. A single unit of the mixer.

channel. Equation (2) can be spanned into three-dimensional autonomous system by setting $z=\omega t$, with a Jacobian matrix $J$ :

$J=\left[\begin{array}{ccc}0 & -2 v_{0} y_{0} / w_{m 2}^{2} & 0 \\ -2 v_{p}\left(x-x_{c}\right) \sin z / w_{s 2}^{2} & 0 & v_{p} \omega\left\{1-\left[\left(x-x_{c}\right) / w_{s 2}\right]^{2}\right\} \cos z \\ 0 & 0 & 0\end{array}\right]$.

Eigenvalues of $J$ corresponding to the equilibrium point B with the coordinate $\left(0, w_{m}\right)$ are

$$
\lambda_{1}=0 \quad \lambda_{2,3}= \pm \sqrt{-v_{0} v_{p} \sin z} .
$$

Therefore, if $\sin z<0, \mathrm{~B}$ is a saddle point. While if $\sin z>0$, $\mathrm{B}$ is a center point.

Eigenvectors of these eigenvalues dictate the manifolds of this EP. Because $z$ increases constantly, B appears as a saddle point and a center point alternately. The manifolds of B also vary with time. The other EP of (2) have a similar stability character with B. All the manifolds of these four EPs add up to the whole vector field in the channel. Through this kind of alternate changes from saddle to center and vice versa, these corners can stretch and fold the fluid elements adjacent to them. Figure 3 shows this kind of stretching and folding of a small volume of fluid in the channel. After about $20 \mathrm{~s}$ the rectangular volume in the beginning turns into a complicated pattern. Therefore in this system there does exist a set of stretching and folding that is needed for the chaotic mixing in the channel.

\section{Poincaré mapping analysis}

Typically, there are two classes of mixers, the closed flow system and the open flow system. Mathematically, the closed flow system corresponds to an initial value problem, with simple boundary conditions, and the motion of particles will be bounded. For an open flow system such as a tubular device, square wave, serpentine mixer or twisted curve pipe, the boundary conditions are time independent, a mapping or a Poincaré section for each geometric symmetry unit can be defined to project the stretching and folding to a cross section. The mapping can considerably simplify analysis by means of a reduction in the number of dimensions, as well as confine the open flow to a bounded area.

For an open flow system governed by (1), with time dependent boundary conditions, this method can still be valid by setting an appropriate mapping. First, set $z=\omega t$, span (1) into an autonomous system. For the trajectory of any initial point, choose Poincaré section to be $x_{n}=$ $n \times p c h, n=1,2, \ldots$, the trajectory will intersect these planes successively at points $P_{1}, P_{2}, \ldots, P_{n} \ldots$, and define a mapping $P_{n+1}=\Phi\left(P_{n}\right)$. With this mapping, $z$ will go 


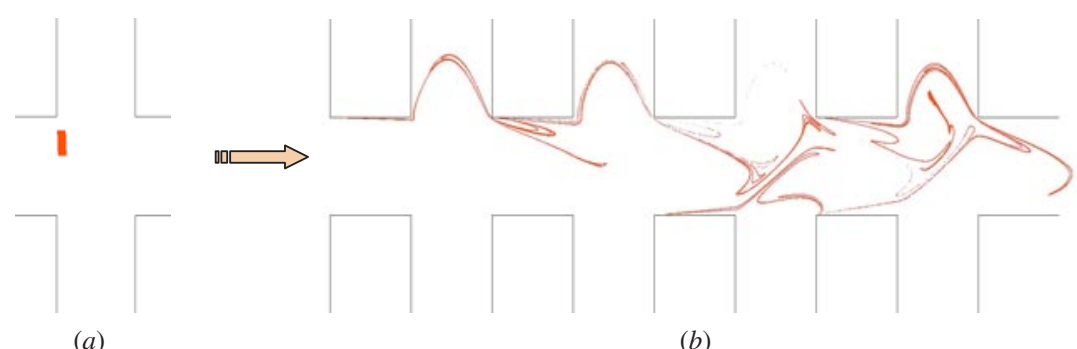

(a)

(b)

Figure 3. Stretching and folding of a small volume of fluid. (a) Initial particle location $(b)$ fluid having been stretched and folded.

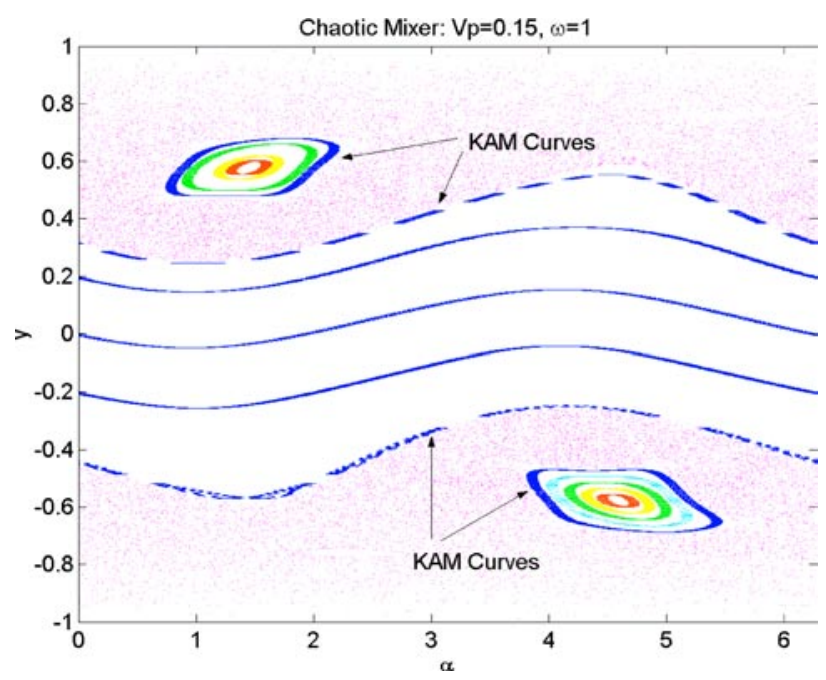

(a)

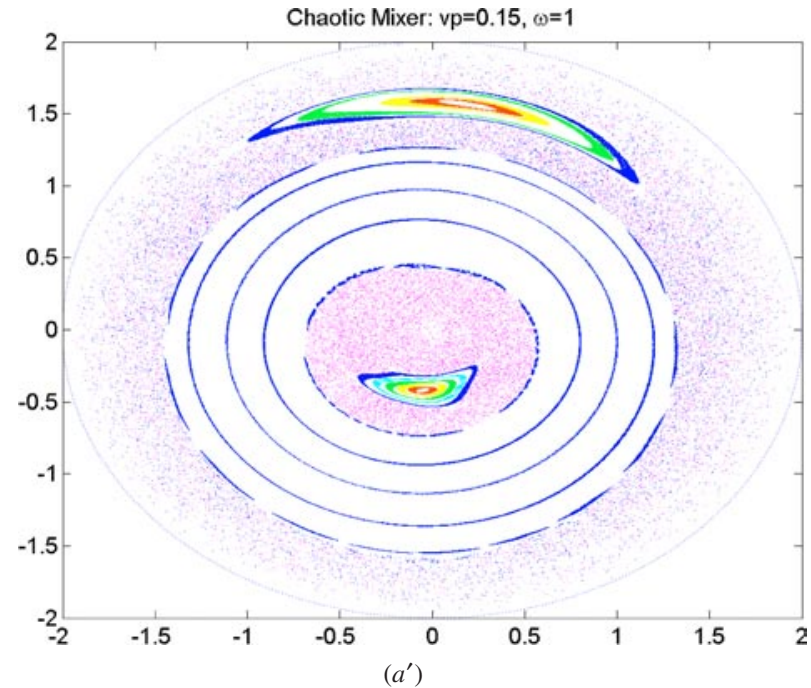

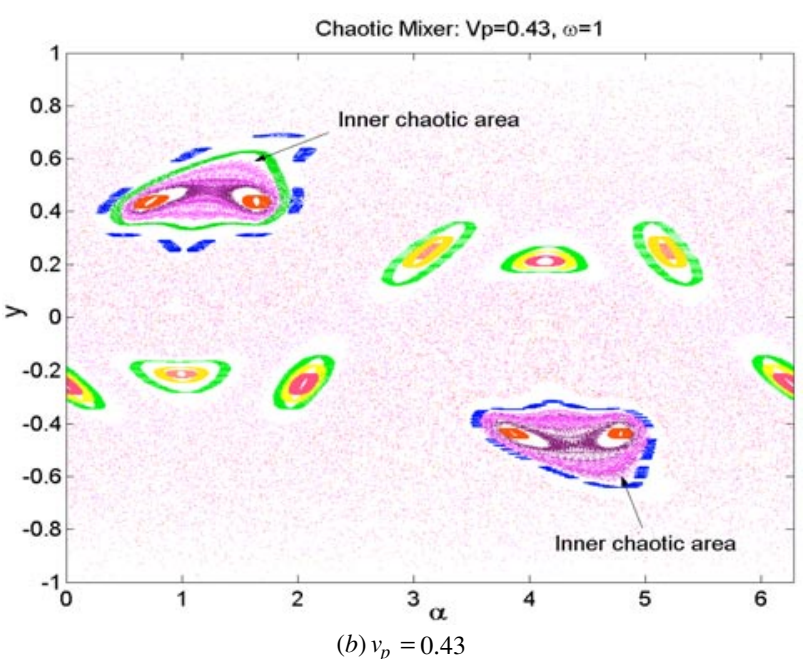

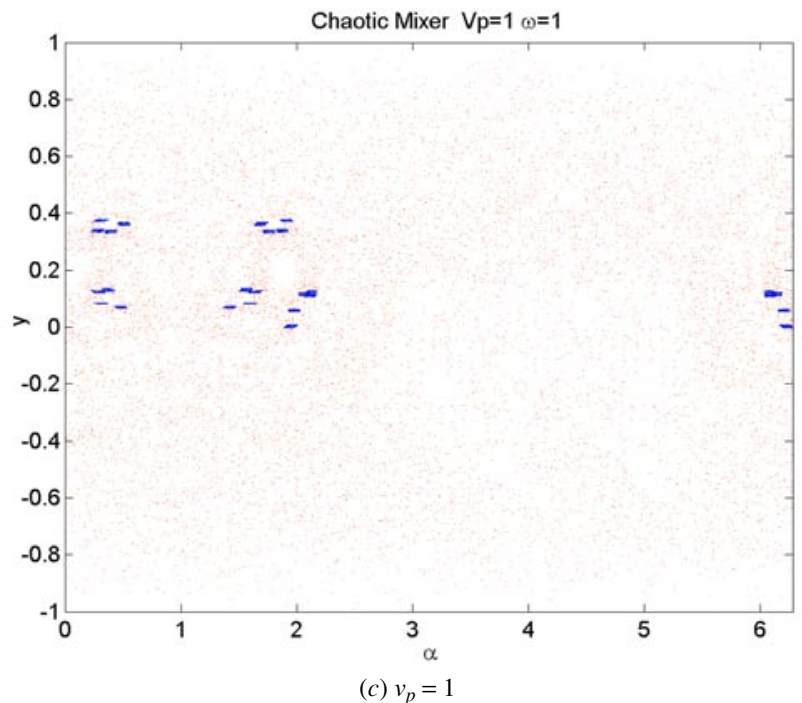

(c) $v_{p}=1$

Figure 4. Poincaré sections of the chaotic mixer for different values of $v_{p}, 0.15,0.43$ and 1 in $(a),(b)$ and $(c)$, respectively. The Poincare section $\left(a^{\prime}\right)$ is a variation of the mapping formula: $(y n+1)$ as radius, $\alpha$ as angle, draw in polar coordinate. This mapping has the advantage of showing a continuation of angle $\alpha$ (also means time).

to infinity as $t$ keeps increasing. Since $z$ only appears in the autonomous system as a term of the sinusoidal function, we can define a new variable $\alpha=\operatorname{modular}(z, 2 \pi)$. Substituting $z$ with $\alpha$ in the original system will not change its dynamic characteristics. Therefore we have set a new mapping for $P_{n}\left(y_{n}, \alpha_{n}\right)$. Because (1) is a strong nonlinear system, we cannot get an explicit formula of that mapping. Each point can be calculated by integrating (1) to the next Poincaré section plane.

Figure 4 depicts Poincaré sections of the chaotic mixer for different amplitudes of perturbation. In this group of figures, the dotted areas are the chaotic areas. A point in this area will have the possibility to arrive at any place within this area. While the areas of circles and lines denote quasi-periodic 
motions or torus; particles here can only move in a periodic or quasi-periodic way. The curves that separate the periodic area from the chaotic area are called KAM curves [10]. Any trajectory of an initial point in one attractor (the quasi periodic area or the chaotic area) cannot be transferred from the KAM curves to another attractor. Therefore these KAM curves act as boundaries confining mixing of fluid elements with those in other areas.

When $v_{p}=0$, all elements in the channel will move in a quasi-periodic way. The Poincaré sections can only have parallel straight lines. As $v_{p}$ increases, these parallel straight lines turn into wave lines, and the outer lines will break up into chaotic areas. In figure $4(a)$, when $v_{p}$ increases to 0.15 , a large portion of elements still continue to move periodically. Only particles close to the channel walls will turn to chaos. But we can see that the chaotic areas are not uniform; there are small periodic areas encircled in them. As the perturbation amplitude increases, the outer KAM curves will break up further and the big periodic area close to the interface in figure 4(a) will shrink gradually and then separate to a chain of periodic islands. This chain acts as a barrier separating the upper and lower chaotic areas, even though the KAM curves of the upper and lower chaotic areas are almost identical. When $v_{p}$ increase beyond this critical value (about 0.42 ), this chain will separate, and chaos pathways will appear among these islands. Chaotic fluid elements on one side of the channel can pass these pathways to the other side as shown in figure $4(b)$, in which $v_{p}=0.43$. As $v_{p}$ increases further, this chain of separated islands will shrink and each one will break into several small islands till all of them are merged by the chaotic areas.

For the areas encircled by the KAM curves close to the channel wall in figure $4(b)$, in the process of breaking up of the KAM curves and area separating, we can find that some inner chaotic areas appear and then become connected with the outer chaotic area as the breaking up of the outer KAM curves. Before breaking up, although particles in these inner chaotic areas move in a chaotic regime, the stretching and folding are confined to a very limited area, and good mixing cannot be achieved in these areas because most of these inner chaotic areas lie on one side of the interface line. These inner chaotic areas also include some periodic areas. This kind of nesting of multiple layers is a prominent character of nonintegrable Hamiltonian systems.

When $v_{p}$ increases to 1 , almost all the mapping areas become chaotic as shown in figure $4(c)$, and a good mixing can be expected.

We can trace back from the definition of the mapping to learn more about how these Poincaré sections can contribute to our understanding of mixing. An area encircled by the KAM curve can be mapped to a continuous area in the beginning channel of the mixer as shown in figure 5. Therefore fluid in the left area will never mix with fluid in the other areas of the beginning channel.

Time trajectories and fast Fourier transform results for an initial point in a quasi-periodic attractor and a chaotic attractor are shown in figure 6. Chaotic motion has continuous spectra in a wide span of frequencies, while quasi-periodic motion appears in a more regular way [18].

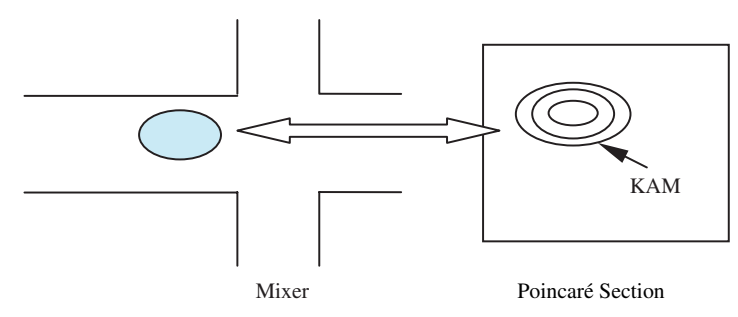

Figure 5. Schematic showing the mapping between KAM curve in the Poincaré section and area with quasi-periodic motion in the beginning channel.

\section{Lyapunov exponents and optimization of the system with multiple side channels}

The Poincaré sections analyzed in section 3 can systematically reduce the number of dimensions of the system, and illustrate the system's route to chaos: breaking up of the KAM curves and the shrinking of the quasi-periodic areas. From the viewpoint of practical mixer design, we still want to know how the parameters of the perturbation contribute to the chaos in the microchannel. Although the Poincaré sections can supply us with some information about the $y_{0}$ and $t_{0}$ to chaos, using it further to reduce the system to a lower dimension is not as applicable as in some traditional high-dimensional cases [10]; moreover, Poincaré sections can only give us schematic illustrations. Lyapunov exponents (LE), as the average exponential rates of divergence or convergence of nearby orbits in the phase space, can describe chaos in a quantitative manner, and therefore, can be used to measure and analyze the relationships of different parameters with chaos. By choosing appropriate parameters based on the LE results, we can further optimize the design and operation of the microchannel.

Equation (1), as a continuous time system, can be rewritten as $\dot{x}=f(x, t)$. For any initial condition $x_{0} \in R^{n}$, the LE of $x_{0}$ are

$$
\lambda_{i}=\lim _{t \rightarrow \infty} \frac{1}{t} \ln \left|m_{i}(t)\right| \quad i=1, \ldots, n
$$

whenever the limit exists. Where $m_{1}(t), m_{2}(t)$ to $m_{n}(t)$ are the eigenvalues of fundamental solution matrix $\Phi_{t}\left(x_{0}\right)$, satisfying

$$
\dot{\Phi}_{t}=D f(x, t) \Phi .
$$

Therefore, LEs are a generalization of the eigenvalues at an equilibrium point. They can be used to determine the stability of equilibrium points, periodic solutions, quasiperiodic and chaotic behavior. Because the channel flow is a Hamiltonian system, the autonomous system spanned by (1) can only have two groups of LE: $(0,0,0),(+\lambda, 0,-\lambda), \lambda$ is a positive real number. The first group corresponds to periodic or quasi-periodic solutions. The second group corresponds to chaos. We can determine whether it is chaotic by judging the maximal LE.

Two different algorithms are used to double-check our numerical results $[12,13,15]$. The convergence of the maximal LE for different steps of iterations is shown in figure 7. LE will converge after about 80 million iterations.

From Poincare sections in figure 4, we know that the trajectory of any initial point will be trapped in the attractor that the initial point lies in, whether it is a chaotic attractor 


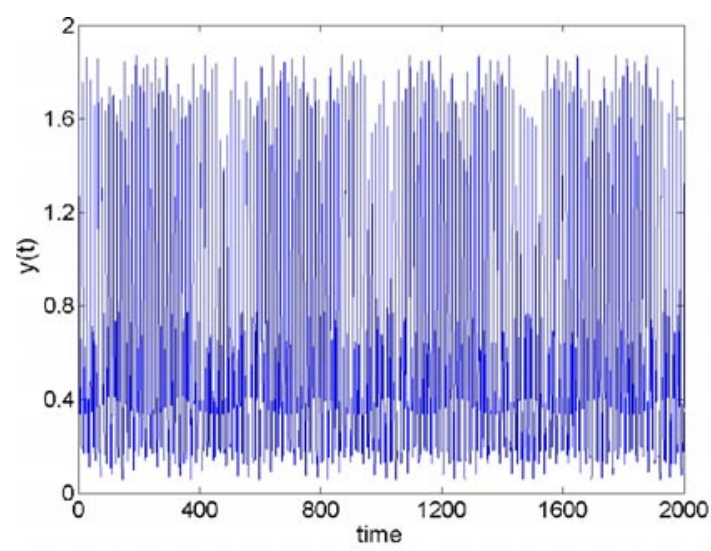

(a1) Time trajectory

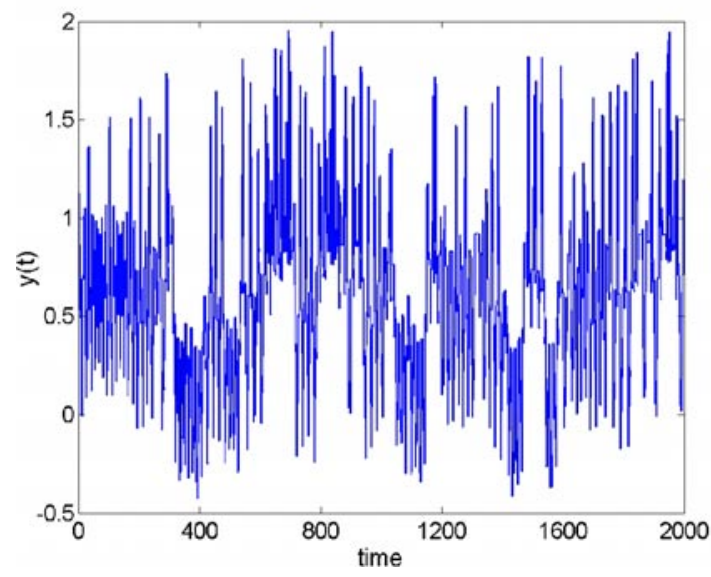

(b1) Time trajectory

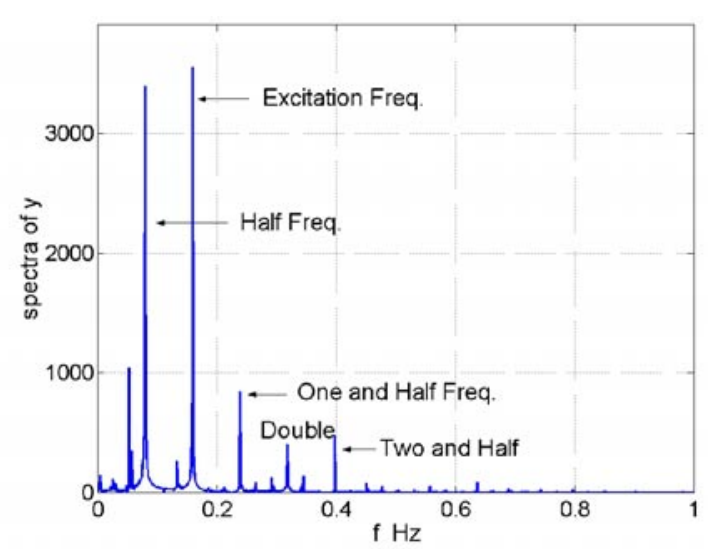

(a2) FFT

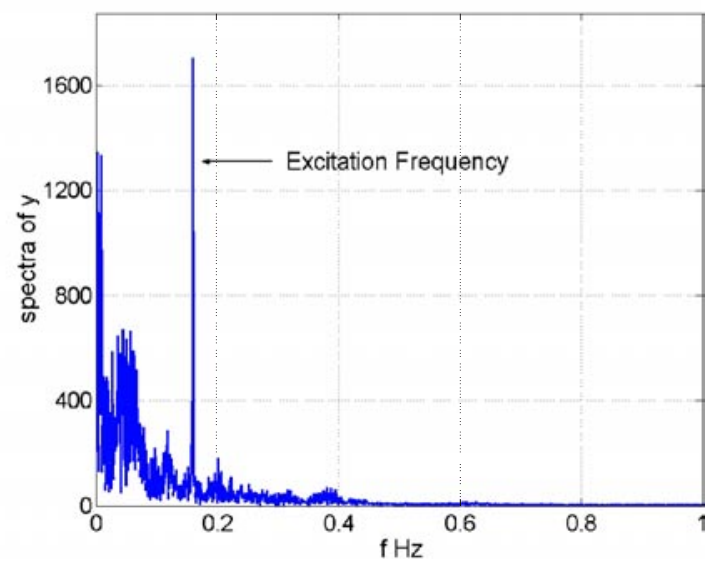

(b2) FFT

Figure 6. Contrast of trajectories and FFT for the same initial point $[0,0.15]$ for different values of $v_{p}$. In $(a), v_{p}=0.9$, and in $(b), v_{p}=0.6$.

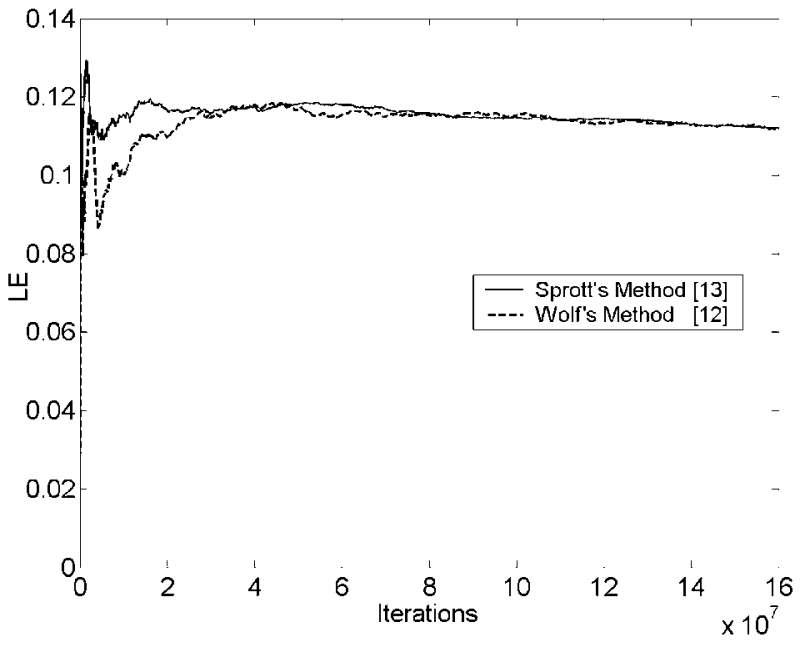

Figure 7. The convergence of the maximum Lypaunov exponent (initial point $[0,0], v_{p} / v_{0}=1, \omega=1.1$ ).

or a quasi periodic attractor. Therefore, the LE should also depend on the initial values, which is quite different from simple temporal chaotic systems such as the Lorenz attractor. To investigate the effect of the amplitude of perturbation to chaos, we calculate the maximal LE for different values of $v_{p}$ and $y_{0}\left(x_{0}=0, t_{0}=0\right)$ by dividing the $v_{p}$ and $y_{0}$ plane into grids to obtain figure 8 . In figure 8 , the LE in the blue areas is zero, while in the other areas they are positive. In the chaotic areas with a positive number, the LE will increase as $v_{p}$ increases, when $v_{p}=1$, LE is about 0.12 (but varies with different $y_{0}$ in small amplitude). The frequency of perturbation is also an important parameter. Figure 9 is a $3 \mathrm{D}$ plot of LE versus $v_{p}$ and $\omega$. We can see that when $\omega$ is higher than about 3.5 , the LE will decrease to zero for the initial points close to the interface line. For most of the initial points, $\omega \approx 1.4$ is the best frequency. For the perturbation amplitude, $v_{p}$ cannot be increased to a very high value at the same time; this is because if $v_{p}$ is big, the pump sources will need more power to disturb the fluid from the side channels. Meanwhile, the mean velocity downstream of the main channel will decrease significantly.

Figure 10 gives out the LE plot of the particles in the beginning channel. In this case, we choose $\omega=1.4$ and $v_{p}=$ 1 to be the optimal parameters of the perturbation. The parabolic line in figure 10 stands for $x=-v_{0}\left(1-y^{2}\right) T$, $T$ is the period of the perturbation. This line and the $x=0$ line compose a closed boundary. All the fluid particles in the area encircled by this boundary will come into the main channel in the next period. In this area, the average of LE is 0.13 and the standard deviation is 0.02 , and only very few small zero LE areas exist, which means we can get a very good mixing for almost all the fluid elements. Due to spatial symmetry, the fluid coming in the periods following will have the same LE values and patterns as in this area, therefore we 


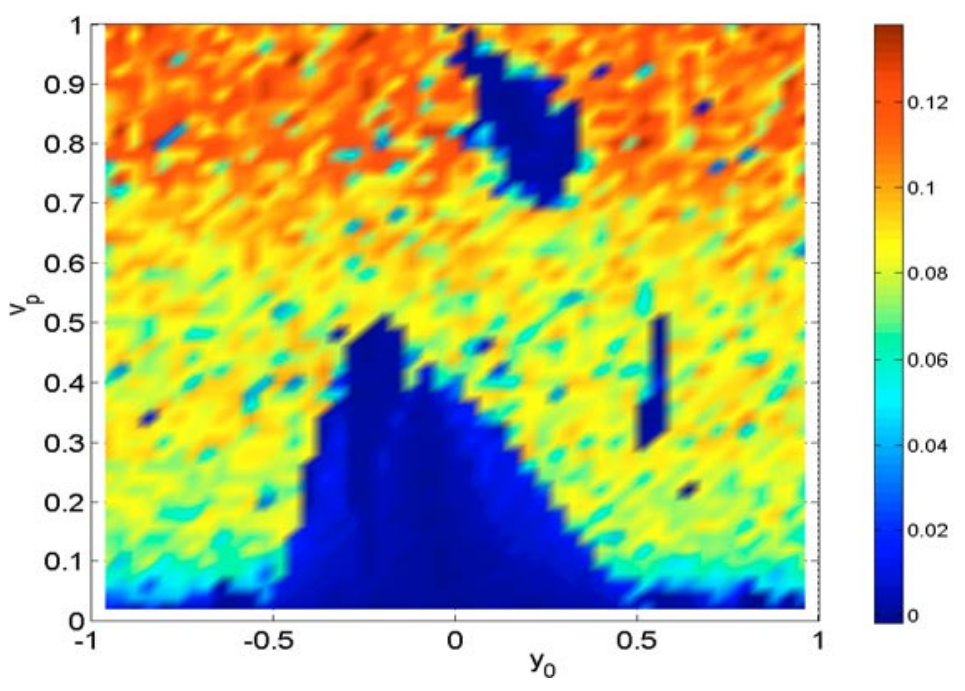

Figure 8. The contour plot of Lypaunov exponent in $y_{0}-v_{p}$ plane.

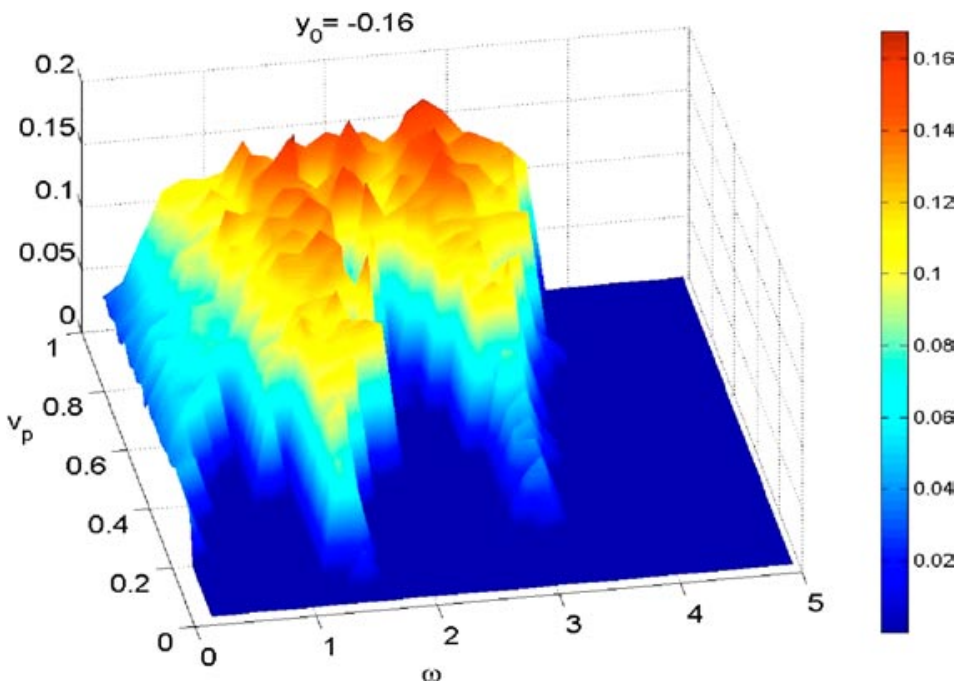

Figure 9. 3D plot of Lypaunov exponents as a function of $v_{p}$ and $\omega$, the initial point of LE is $(0,-0.16)$.

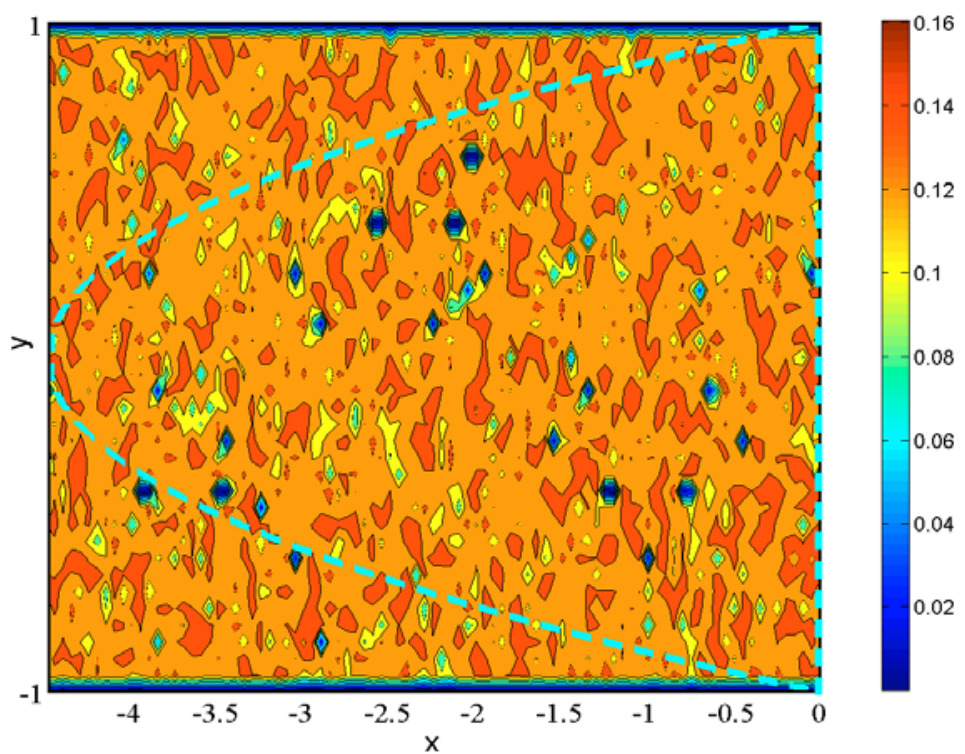

Figure 10. The contour plot of Lypaunov exponent for one period of fluid elements in the beginning channel. 
Table 1. Minimal length of mixing for some important biomolecule solutions (LE is set to be 0.1).

\begin{tabular}{lrllll}
\hline $\begin{array}{l}\text { Diffusing } \\
\text { particle } \\
\text { in water }\end{array}$ & $\begin{array}{l}\text { Molecular } \\
\text { weight } \\
(\mathrm{g} \text { mole }\end{array}$ & $\begin{array}{l}\text { Diffusion } \\
\text { coefficient } \\
D\left(\mathrm{~m}^{2} \mathrm{~s}^{-1}\right)\end{array}$ & $\begin{array}{l}\text { Minimum length } \\
\text { for mixing }(\mu \mathrm{m})\end{array}$ & $\begin{array}{l}\text { Minimum units } \\
\text { for mixing } \\
\text { (number of pairs of } \\
\text { side channels) }\end{array}$ & $\begin{array}{l}\text { Length purely } \\
\text { dependent on } \\
\text { diffusion }(\mu \mathrm{m})\end{array}$ \\
\hline $\begin{array}{l}\text { Hemoglobin } \\
\text { Viral DNA }\end{array}$ & 68000 & $7.3 \times 10^{-11}$ & 3202 & 5 & $2.19 \times 10^{5}$ \\
T7 Bacteriophage & 375000000 & $1.4 \times 10^{-12}$ & 5880 & 8 & $1.14 \times 10^{7}$ \\
$\begin{array}{l}\text { Red Blood Cell } \\
(\sim 5.6 \mu \mathrm{m})\end{array}$ & $\sim 6 \times 10^{13}$ & $6.8 \times 10^{-14}$ & 8050 & 6 & $1.68 \times 10^{6}$ \\
\hline
\end{tabular}

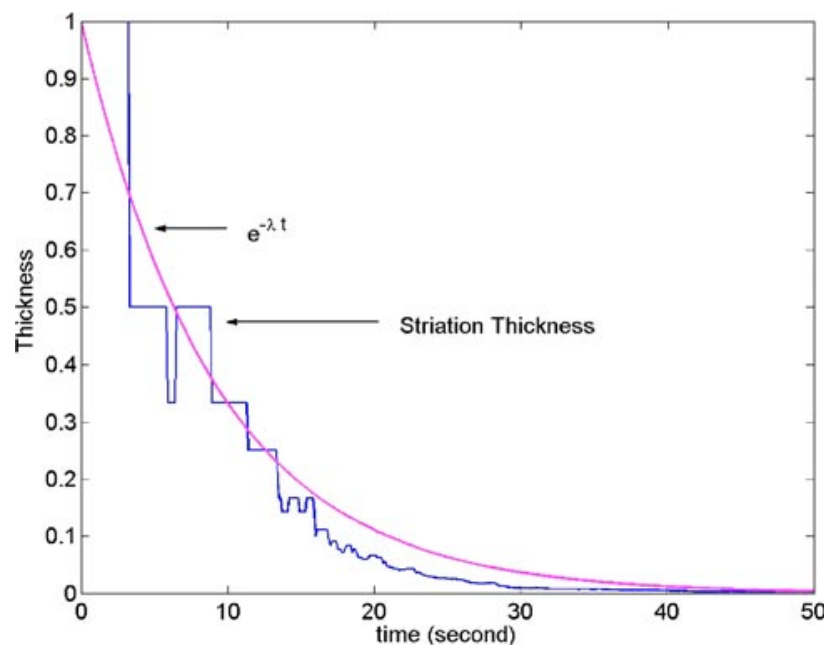

Figure 11. Correlation of the mean striation thickness with the exponential rate of decrease for the maximum Lyapunov exponent.

can forecast mixing effects for all the liquid to be mixed in the channel.

From figures 8-10, we know that the LE is closely related to $v_{p}, \omega, y_{0}$ and $t_{0}$, and the system can be called a spatial temporal chaotic mixer. If the optimal values for $v_{p}$ and $\omega$ can be chosen in the operation, then most of the liquid in the channel can be well mixed. For the tiny separated quasi-periodic areas, diffusion is very easy to achieve because of their small dimensions.

\section{Minimum micromixer length to complete mixing}

Diffusion effect of different fluids in the channel will be taken into account in this part to help determine the minimum channel length needed in the micro mixer. The minimum diffusion length, $x_{m}$, for a complete mixing of two stream fluids can be determined $[1,9]$ by

$$
x_{m}=y_{d}^{2} \frac{v_{a}}{2 D}
$$

where $v_{a}$ is the average speed of the fluid in the mixer, $y_{d}$ is the breadth of two stream fluid in the span wise direction and $D$ is molecular diffusivity.

Note that $v_{a}$ in the main channel is smaller than $v_{0}$, and can be expressed as $v_{a}=\delta v_{0}, \delta \in[0,1]$ can be determined by transverse perturbation numerically. The breadth $y_{d}$, equivalent to the average molecular diffusion distance, can be shown to be equal to the striation thickness of the layers of the fluids in the process of stretching and folding [10]. For any

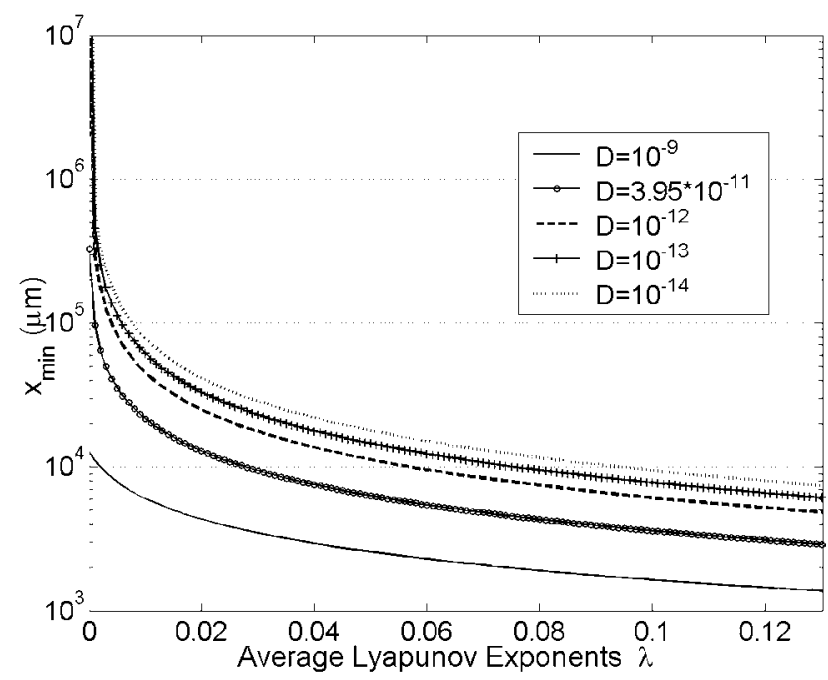

Figure 12. Minimum length needed for fully mixing in different Lypaunov exponent and molecular diffusivity $D$. The unit of $D$ in this figure is $\mathrm{m}^{2} / \mathrm{s}$.

initial point in the chaotic area, the mean striation thickness along its trajectory is calculated, and the decrease in striation thickness is consistent with the exponential rate of decrease for the maximum LE as shown in figure 11. Therefore $y_{d}$ can be related to the maximal LE [16] by

$$
y_{d}=\omega_{m} \mathrm{e}^{-\lambda t} \text {. }
$$

$D$ can be determined by the property of the fluid. For the diluted water solution of sucrose in the channel [5],

$$
D=3.95 \times 10^{-11} \mathrm{~m}^{2} \mathrm{~s}^{-1} .
$$

Substituting $v_{a},(5)$ and $D$ into (4), we get

$$
x_{m}=w_{m}^{2} \mathrm{e}^{-2 \lambda t} \frac{\delta v_{0}}{2 D} .
$$

Note that $x_{m}=\delta v_{0} t \Rightarrow t=x_{m} / \delta v_{0}$, after we choose $\delta=0.8$, $w_{m}=400 \mu \mathrm{m}, v_{0}=200 \mu \mathrm{m} \mathrm{s}^{-1}$ and $\lambda$, and let the average Lyapunov exponents be 0.1 , we get

$$
x_{m} \approx 3600 \mu \mathrm{m}<5 \text { units. }
$$

Therefore after five units, namely five pairs of side channels in figure 1(b), from the starting point in the channel, most of the fluids are well mixed. While if there is no active pump, which means $\lambda=0$ and $\delta=1$, the minimum length of diffusion for the channel is

$$
x_{m}^{\prime}=w_{m}^{2} v_{0} / 2 D=405036 \mu \mathrm{m} \approx 40 \mathrm{~cm} .
$$


We can see that the chaotic mixer can decrease the diffusion-based mixing length of the micro-channel by two orders of magnitude. The minimum channel length of the chaotic mixer determined by this LE approach is consistent with the experimental result in [5] and can be seen as an upper bound in this kind of mixer design.

The variation of the minimum diffusion length of the chaotic mixer versus the average Lyapunov exponents in different molecular diffusivity is given in figure 12 .

For some other important biomolecules suspended in water at $310 \mathrm{~K}$ [17], their minimal length and minimal units needed for a full mixing in this micro channel are listed in table 1 . We can see that not more than $1 \mathrm{~cm}$ of this micro channel is needed even for Red Blood Cell (red blood cell) solutions with a small diffusion coefficient.

\section{Conclusion}

Based on the concepts of efficient stretching and folding of fluid in microchannels, an active micro mixer is designed and investigated using chaotic dynamics. The governing equations are derived to study dynamic behaviors of the system. Poincaré sections are used to reveal the route of the system from quasiperiodic to chaos. Because of transverse perturbation from the pressure driven sources, the outer KAM curves will break up; the chaotic areas will expand and quasi-periodic areas will shrink with an increase in perturbation. Lyapunov exponents are used to measure the strength of those chaotic behaviors and then can be used to optimize the design and operation. The Lyapunov exponents calculated from the two different numerical methods are consistent. The fact that the calculated exponents depend on initial conditions makes this spatialtemporal chaotic system different from traditional simple chaos (Lorenz attractor). By using the optimal parameters, almost all the fluid in the channel can be well mixed. The minimum channel length for a full mixing can be estimated and is much smaller than that in the passive mixer design.

It is interesting to note that this spatial-temporal chaotic mixer can have similar Poincaré sections with some spatial serpentine devices [8] by a suitable mapping. Using the combination of the Poincaré section and Lyapunov exponent analysis, we have studied the chaotic characteristics of the system thoroughly. Poincaré sections can explain the process of the system from periodic or quasi-periodic to chaos, while LE can supply information of the effects of the parameters to chaos and can also be used for optimization.

Note that the pressure driven sources of perturbation can also be achieved by an electric field; the method used and the results in this paper can also be used in the design of electrokinetically driven micro mixers. Further experimental studies in the temporal three-dimensional chaotic mixers driven and stirred by pressure or an electric field are still needed.

\section{Acknowledgments}

The authors would like to thank Professor Tongxi Yu, Professor Yitshak Zohar and Shilun Ruan at HKUST. The help of computer programming from Dr Yuen-Chang Sun in GigaMedia Inc., Taiwan is greatly appreciated as well. This work is supported by the Institute of Integrated Microsystem, HKUST (contract no I2MS01/02.EG08).

\section{References}

[1] Stroock A D, Dertinger S K W, Ajdari A, Mezic I, Stone H A and Whitesides G M 2002 Chaotic mixer for microchannels Science 295 647-51

[2] Stroock A D, Dertinger S K W, Whitesides G M and Ajdari A 2002 Patterning flows using grooved surfaces Anal. Chem. $745306-12$

[3] Gobby D, Angeli P and Gavriilidis A 2001 Mixing characteristics of T-type microfluidic mixers J. Micromech. Microeng. 11 126-32

[4] Johnson T J, Ross D and Locascio L E 2002 Rapid microfluidic mixing Anal. Chem. 74 45-51

[5] Lee Y K, Deval J, Tabeling P and Ho C M 2001 Chaotic mixing in electrokinetically and pressure driven micro flows Proc. 14th IEEE Workshop on Micro Electro Mechanical Systems (Interlaken, Switzerland, Jan.) pp 483-6

[6] Mingqiang Yi and Bau H H 2000 The kinematics of bend-induced stirring in micro-conduits Proc. ASME Intl. Mechanical Engineering Congress and Exposition, MEMS vol 2 pp 489-96

[7] Evans J, Liepmann D and Pisano A P 1997 Planar laminar mixer Proc. 10th IEEE Workshop on Micro Electro Mechanical Systems (MEMS '97) (Nagoya, Japan, Jan.) pp 96-101

[8] Liu R H, Stremler M A, Sharp K V, Olsen M G, Santiago J G, Adrian R J, Aref H B and David J 2000 Passive mixing in a three-dimensional serpentine microchannel J. MEMS 9 190-7

[9] Bessoth F G, deMello A J and Manz A 1999 Microstructure for efficient continuous flow mixing Anal. Commun. 36 213-5

[10] Ottino J M 1989 The Kinematics of Mixing: stretching, Chaos, and Transport (New York: Cambridge University Press)

[11] Aref H 1984 Stirring by chaotic advection J. Fluid Mech. 143 $1-21$

[12] Wolf A, Swift J B, Swinney H L and Vastano J A 1985 Determining Lyapunov exponents from a time series Physica D 16 285-317

[13] Sprott J C 2000 Numerical calculation of largest Lyapunov exponent http://sprott.physics. wisc.edu/chaos/lyapexp.htm

[14] Volpert M, Meinhart C D, Mezic I and Dahelh M 1999 An actively controlled micromixer Proc. ASME Intl. Mechanical Engineering Congress and Exposition, MEMS (Nashville, TN, Nov.) pp 483-7

[15] Lee Y K 2002 Lyapunov exponents of a micro chaotic mixer Intl. J. Nonlinear Sci. Numer. Simul. 3 561-3

[16] Tang X Z and Boozer A H 1999 Design criteria of a chemical reactor based on a chaotic flow Chaos 9 183-94

[17] Freitas R A 1999 Nanomedicine vol 1 (Austin, TX: Landes Bioscience)

[18] Tufillaro N B, Abbott T and Reilly J 1992 An Experimental Approach to Nonlinear Dynamics and Chaos (Redwood City, CA: Addision-Wesley)

[19] Volpert M, Meinhart C, Mezic I and Dahleh M 2002 Modeling and numerical analysis of mixing in an actively controlled micromixer HEFAT2002: 1st Intl. Conf. on Heat Transfer, Fluid Mechanics, and Thermodynamics (Kruger Park, South Africa, 8-10 April 2002)

[20] Desmukh A A, Liepmann D and Pisano A P 2000 Continuous micromixer with pulsatile micropumps Solid-State Sensor and Actuator Workshop (Hilton Head Island, SC, USA, 4-8 June 2000) 